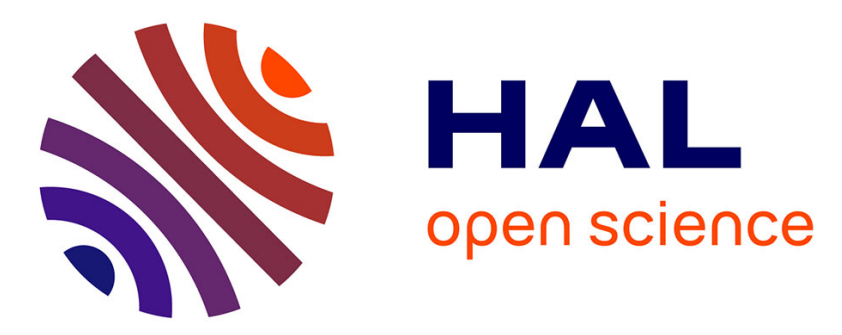

\title{
Visual Servoing Schemes for Automatic Nanopositioning Under Scanning Electron Microscope.
}

\author{
Naresh Marturi, Brahim Tamadazte, Sounkalo Dembélé, Nadine Piat
}

\section{To cite this version:}

Naresh Marturi, Brahim Tamadazte, Sounkalo Dembélé, Nadine Piat. Visual Servoing Schemes for Automatic Nanopositioning Under Scanning Electron Microscope.. IEEE International Conference on Robotics and Automation, ICRA'2014., Jan 2014, Hong Kong SAR China. pp.1-6. hal-01051592

\section{HAL Id: hal-01051592 https://hal.science/hal-01051592}

Submitted on 25 Jul 2014

HAL is a multi-disciplinary open access archive for the deposit and dissemination of scientific research documents, whether they are published or not. The documents may come from teaching and research institutions in France or abroad, or from public or private research centers.
L'archive ouverte pluridisciplinaire $\mathbf{H A L}$, est destinée au dépôt et à la diffusion de documents scientifiques de niveau recherche, publiés ou non, émanant des établissements d'enseignement et de recherche français ou étrangers, des laboratoires publics ou privés. 


\title{
Visual Servoing Schemes for Automatic Nanopositioning Under Scanning Electron Microscope
}

\author{
Naresh Marturi, Brahim Tamadazte, Sounkalo Dembélé, and Nadine Piat
}

\begin{abstract}
This paper presents two visual servoing approaches for nanopositioning in a scanning electron microscope (SEM). The first approach uses the total pixel intensities of an image as visual measurements for designing the control law. The positioning error and the platform control are directly linked with the intensity variations. The second approach is a frequency domain method that uses Fourier transform to compute the relative motion between images. In this case, the control law is designed to minimize the error i.e. the 2D motion between current and desired images by controlling the positioning platform movement. Both methods are validated at different experimental conditions for a task of positioning silicon microparts using a piezo-positioning platform. The obtained results demonstrate the efficiency and robustness of the developed methods.
\end{abstract}

\section{INTRODUCTION}

Over the past couple of decades, nanomanipulation has gained significant attention due to the advances in nanoscience and nanotechnology. It is widely used in many industrial and scientific works for handling micronanostructures in order to perform dynamic analysis and characterization of their structural, mechanical, electrical or optical properties. Moreover, manipulation of nanometric objects also benefit in building complex nanoelectromechanical systems (NEMS) [1]. The consequence of this strong demand is the emergence of an active research area concerning the development of assembly and handling technologies on a micro-nanoscale. So far, great progress has already been realized in the development of microassembly stations [2], microgrippers [3], precise manipulation systems as well as robust control strategies. In order to perform nanomanipulation, the basic tasks include identification, positioning and handling of the objects. Out of all, positioning is more challenging at this particular scale mainly due to the lack of accurate feedback information. This problem can be resolved by considering microscopic imaging systems such as SEM [4], transmission electron microscope (TEM) or atomic force microscope (AFM) as the underlying sensors to control the behavior of robotic devices during the process. With its ability of producing images with high resolution at high magnification in real-time, a SEM is always favoured

This work is conducted with a financial support from the project NANOROBUST (ANR- 11-NANO-006) funded by the Agence Nationale de la Recherche (ANR), France. It is also performed in the framework of the Labex ACTION (ANR-11-LABEX-01-01) and the Equipex ROBOTEX (ANR-10-EQPX-44-01) projects.

Naresh Marturi, Brahim Tamadazte, Sounkalo Dembélé, and Nadine Piat are with Automatic control and Micro Mechatronic Systems (AS2M) department, Institute FEMTO-ST, Besançon, France. naresh.marturi at femto-st.fr as an imaging tool for automatic nanomanipulation applications.

In general, the method of controlling the robotic devices using vision feedback is commonly termed as visual servoing [5]. The basic visual servoing approaches are classified in two types: position-based and image-based. For positionbased visual servoing, 3D pose of the robot is derived from the images and is used to minimize the error between observed and reference poses in the cartesian space [6]. Whereas for image-based visual servoing, the control strategy is based on minimizing the error between current and reference features observed in the images [7]. These features are local geometric contents (i.e. edges, corners, etc.) of an object or specially used fiducial markers and are extracted from visual tracking. This tracking process plays an essential role in the design of visual servoing strategies. However, recent developments have shown that this tracking process for visual servoing can be completely replaced by using the global image information like pixel intensities [8] or image entropy. Also, as the total image information is used, it increases the robustness in minimizing the error and simultaneously increases the overall accuracy of the system because of the redundancy information.

With SEM as an imaging tool, acquiring images to use them with visual servoing is always a challenging task. This is mainly due to the addition of huge amount of noise during image acquisition process at higher scan rates. Moreover, the pixels are acquired one at a time slowing down the overall acquisition rate. Apart from this, when the objects are in motion, the images appeared to be distorted because of the sequential raster scanning the surface. This phenomenon mainly raises the difficulty in applying any visual tracking algorithms. In order to tackle this problem, in this paper, we consider the direct approach of using the global image information rather than local features for visual servoing. Using this technique, two methods have been implemented for positioning the silicon micro-objects by controlling the motion of the positioning platform. The first one uses the global pixel intensity values and the second one is based on the global motion estimation between images. The developed methods are evaluated at different experimental conditions such as varying scan speed and magnifications.

\section{SET-UP AND MODELLING}

\section{A. Experimental set-up}

The experimental set-up used for this work is shown in the Fig. 1. It consists of a JEOL JSM 820 SEM, an image acquisition system (DISS5 from Point electronic $\mathrm{GmbH}$ ), a 


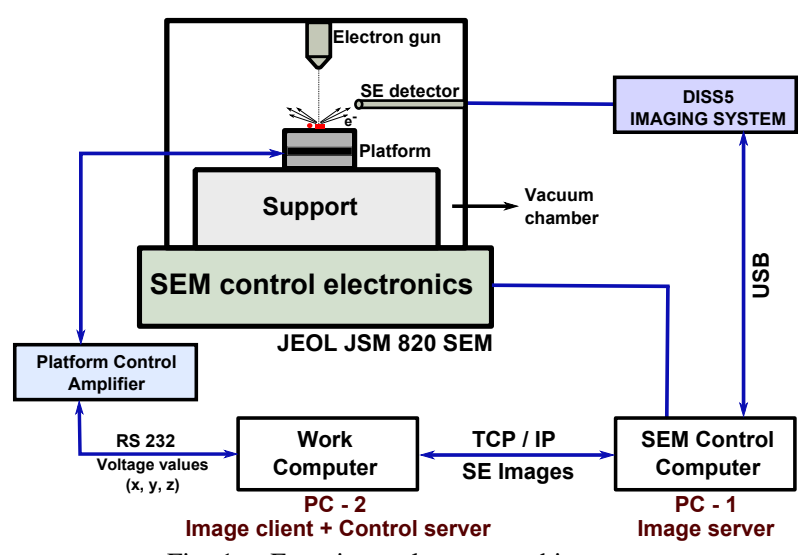

Fig. 1. Experimental set-up architecture.

3 degrees of freedom $(x, y, z)$ open loop piezo positioning platform (TRITOR 100 from Piezosystem Jena $\mathrm{GmbH}$ ) and two computers. The positioning platform is mounted inside SEM vacuum chamber and is controlled using a 3 channel piezo controller NV 40/3. The maximum possible motion on all axes is up to $100 \mu \mathrm{m}$ with a resolution of $0.2 \mathrm{~nm}$. The primary computer (PC 1: Intel Pentium 4, CPU 2.24 $\mathrm{GHz}$ and $512 \mathrm{MB}$ of RAM) is connected to the SEM control electronics and imaging system. It is solely responsible for controlling the microscope. The work computer (PC 2: Intel Core 2 Duo, CPU $3.16 \mathrm{GHz}$, and $3.25 \mathrm{~GB}$ of RAM) is connected to the primary one using an Ethernet cross-over cable. The communication between the two computers is accomplished by implementing a client-server model using TCP/IP. The server program runs continuously from the primary computer and is responsible for receiving and digitizing the data coming from the image acquisition device. Later, the acquired images are transferred to the client upon request. On the work computer, the image client receives these images and transfers them to the control server. The control server computes the required control and issues a voltage command to the platform controller via RS-232 (serial port).

\section{B. Platform voltage-displacement model}

In general, the displacement provided by the positioning platform is a result of the voltage supplied to the piezo actuator. As the control laws, explained in the next section, can only provide the displacements, it is necessary to compute the relationship between displacement and input voltage. Apart from that, it is a well-known fact that the piezoelectric materials exhibit strong nonlinear hysteresis. So, it is also required to compensate this effect beforehand. In order to accomplish this, the experiments are performed by increasing the voltage from initial value $(-19 \mathrm{~V})$ to maximum value $(110 \mathrm{~V})$ with a step change of $1 \mathrm{~V}$ and then decreasing back to the initial value. The respective displacements are measured using a laser interferometer (outside the SEM chamber). The tests are conducted for each axis ( $x$ and $y$ ) separately and the obtained hysteresis curves are shown in Fig. 2(a) and Fig. 2(b). Note that, even though $z$ axis motion can be controlled up to $100 \mu \mathrm{m}$, it is not used in this work since this movement is smaller than the depth of field at the used magnification and cannot be traced. The ascending and

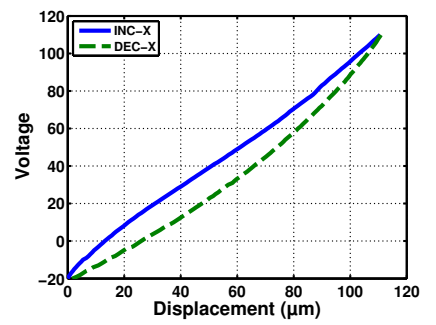

(a)

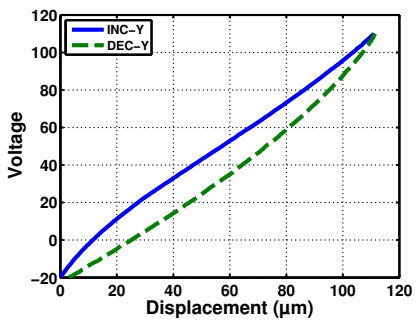

(b)
Fig. 2. Hysteresis curves for (a) $x$-axis (b) $y$-axis of the positioning platform.

descending curves for each axis are approximated by fourth order polynomial given by (1), (2), (3) and (4), respectively. The coefficients of the polynomial are estimated by least squares fit. Finally, the platform control is performed using the displacement vector $\mathbf{d}_{(x, y)}=\left[d_{x} d_{y}\right]^{\top}$ to supply voltages $\left[\begin{array}{ll}V_{x} & V_{y}\end{array}\right]^{\top}$ for the piezo-actuator

$$
\begin{aligned}
& V_{x-i n c}=a_{1 i} d_{x}^{4}+a_{2 i} d_{x}^{3}+a_{3 i} d_{x}^{2}+a_{4 i} d_{x}+a_{5 i} \text { if } \dot{x}>0 \\
& V_{x-\text { dec }}=a_{1 d} d_{x}^{4}+a_{2 d} d_{x}^{3}+a_{3 d} d_{x}^{2}+a_{4 d} d_{x}+a_{5 d} \text { if } \dot{x}<0 \\
& V_{y-i n c}=b_{1 i} d_{y}^{4}+b_{2 i} d_{y}^{3}+b_{3 i} d_{y}^{2}+b_{4 i} d_{y}+b_{5 i} \text { if } \dot{y}>0 \\
& V_{y-\text { dec }}=b_{1 d} d_{y}^{4}+b_{2 d} d_{y}^{3}+b_{3 d} d_{y}^{2}+b_{4 d} d_{y}+b_{5 d} \text { if } \dot{y}>0
\end{aligned}
$$

where, $V_{x}, V_{y}$ and $d_{x}, d_{y}$ are the input voltages and displacements for $x$ and $y$ axes respectively, $a_{k i}, a_{k d}, b_{k i}$ and $b_{k d}$ are the polynomial coefficients for increasing and decreasing curves, $\dot{x}$ and $\dot{y}$ are the change in displacements.

\section{VISUAL SERVOING FOR NANOPOSITIONING}

Considering the problem of using feature tracking for visual servoing in a SEM, in this section we develop two visual servoing methods for nanopositioning. The first method is based on the concept of photometric visual servoing [8] that uses the total image pixel intensities for minimizing the positioning error. The platform control is directly linked with the variation of pixel intensity values in the image. The second method uses the concept of phase correlation in the frequency domain to compute relative motion between two frames. In this case, the control law is designed to minimize the error i.e. the 2D motion between current and desired frames by controlling the platform. Both methods are explained below.

\section{A. Intensity-based visual servoing}

The traditional image-based visual servoing approaches are based on minimizing the error e between current features $\mathbf{s}(t)$ and desired features $\mathbf{s}^{*}$ i.e. $\mathbf{e}=\mathbf{s}(t)-\mathbf{s}^{*}$ [7]. In order to design a vision-based control law, an interaction matrix $\mathbf{L}$ that links the time variation of visual features $\dot{\mathbf{s}}$ with camera instantaneous velocities $\mathbf{v},(\dot{\mathbf{s}}=\mathbf{L v})$ is required. Using this, the final control law is then given by (5).

$$
\mathbf{v}=-\lambda \mathbf{L}^{\dagger} \mathbf{e}
$$

where, $\lambda$ being a positive value to ensure an exponential decrease of the error and $\mathbf{L}^{\dagger}$ is the pseudoinverse of $\mathbf{L}$. 


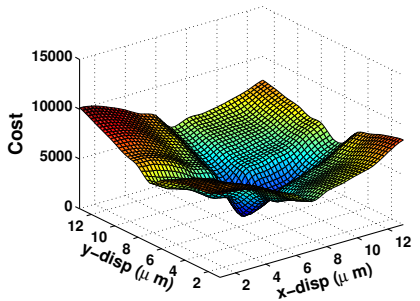

(a)

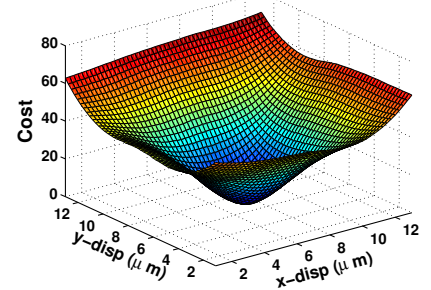

(b)
Fig. 3. Cost functions for (a) intensity-based visual servoing (b) Fourierbased visual servoing.

As mentioned, in this work we use the pixel intensities as the visual features i.e. $\mathbf{s}=\mathbf{I}$. By considering all the pixels in image $f(x, y)$ of size $M \times N$, the visual feature vector is

$$
\mathbf{s}=\mathbf{I}=\left(I_{(1,1)}, I_{(1,2)} \ldots I_{(M, N)}\right)^{\top}
$$

where, $I_{(u, v)}$ is the intensity of a pixel at location $(u, v)$ and $\mathbf{I}$ is a column vector of size $M \times N$. Now, the error is $\mathbf{e}=$ $\mathbf{I}-\mathbf{I}^{*}$. If we consider the problem of error minimization as an optimization problem, the primary goal will be to minimize the cost $C$ given by (7).

$$
C=\mathbf{e}^{\top} \mathbf{e}=\left(\mathbf{I}-\mathbf{I}^{*}\right)^{\boldsymbol{\top}}\left(\mathbf{I}-\mathbf{I}^{*}\right)
$$

When $C$ is minimum, the current position corresponds to the desired position. Fig. 3(a) shows the shape of the cost function that has been computed offline. Now, the interaction matrix $\mathbf{L}_{\mathbf{I}}$ that links the intensity variation with camera instantaneous velocities can be derived by considering the optical flow constraint equation (OFCE) as shown in [9]. For one pixel at $(x, y)$, it can provide a control upto 6 degrees of freedom and is given by

$$
\mathbf{L}_{\mathbf{I}(x, y)}=-\left[\nabla \mathbf{I}_{x} \mathbf{L}_{x}+\nabla \mathbf{I}_{y} \mathbf{L}_{y}\right]_{1 \times 6}
$$

where, $\nabla \mathbf{I}_{x}$ and $\nabla \mathbf{I}_{y}$ are the image gradients. Considering the entire image and using (8), the time variation of visual features is then given by

$$
\dot{\mathbf{I}}=\left[\mathbf{L}_{\mathbf{I}(1,1)} \ldots \mathbf{L}_{\mathbf{I}(M, N)}\right]^{\top} \mathbf{v}
$$

Upon computing the interaction matrix, the control law given by (5) can be used. However, a more feasible solution can be by using a control law derived of the form LevenbergMaquardt optimization technique given by (10) that helps in better convergence [10].

$$
\mathbf{v}=-\lambda^{c} \mathbf{V}_{p}(\mathbf{H}+\mu \operatorname{diag}(\mathbf{H}))^{-1} \mathbf{L}_{\mathbf{I}}^{\top} \mathbf{e}
$$

where, $\lambda$ and $\mu$ are positive gains, ${ }^{c} \mathbf{V}_{p}$ is the transformation matrix from camera frame $\mathcal{R}_{c}$ to platform frame $\mathcal{R}_{p}$ and $\mathbf{H}=\mathbf{L}_{\mathbf{I}}{ }^{\top} \mathbf{L}_{\mathbf{I}}$ is the Hessian matrix. In this work, the gain $\lambda$ is chosen to be adaptive, whose value changes with the variance of error.

Now, the displacement $\mathbf{d}_{(x, y)}$ of the platform is computed using (11).

$$
\mathbf{d}_{(x, y)}=\mathbf{v}_{\text {avg }} t
$$

where, $\mathbf{v}_{\text {avg }}=\frac{\mathbf{v}_{0}+\mathbf{v}_{\text {cur }}}{2}$ is the average velocity, $\mathbf{v}_{0}$ is initial velocity, $\mathbf{v}_{\text {cur }}$ is the current velocity and $t$ is the time taken. For each iteration, the displacement is updated as given by
(12) and the corresponding voltage computed from (1), (2), (3) and (4) is used to move the platform.

$$
\mathbf{d}_{\text {new }}=\mathbf{d}_{\text {prev }}+\mathbf{d}_{\text {cur }}
$$

where, $\mathbf{d}_{n e w}, \mathbf{d}_{\text {prev }}$ and $\mathbf{d}_{\text {cur }}$ are the updated, previous and current displacements, respectively.

\section{B. Fourier-based visual servoing}

This method is based on estimating the motion between the images in the Fourier domain. The main reason for choosing Fourier domain is that with SEM imaging, the brightness and contrast are not constant and it is known that Fourier-based motion estimation is robust to these variations and noise.

1) Motion estimation between images: The translation is estimated using the phase correlation method. It is based on the Fourier shift property which states that the translation in the spatial domain can be seen as the linear phase differences in Fourier domain. Suppose, we have an image $f^{*}(x, y)$ acquired at desired location and $f(x, y)$ is the displaced version of $f^{*}(x, y)$, then

$$
f(x, y)=f^{*}\left(x+\delta_{x}, y+\delta_{y}\right)
$$

Let, $\mathcal{F}^{*}(u, v)$ and $\mathcal{F}(u, v)$ be the Fourier transforms of $f^{*}(x, y)$ and $f(x, y)$, respectively and are computed as

$$
\mathcal{F}(u, v)=\sum_{x=0}^{M-1} \sum_{y=0}^{N-1} f(x, y) e^{-j 2 \pi\left\{\frac{u x}{M}+\frac{v y}{N}\right\}}
$$

According to Fourier shift property

$$
\mathcal{F}(u, v)=\mathcal{F}^{*}(u, v) e^{-j 2 \pi\left\{\frac{u}{M} \delta_{x}+\frac{v}{N} \delta_{y}\right\}}
$$

Now, the translation is reflected in the exponential part. It can be estimated by computing the normalized cross-power spectrum $\widehat{C}(u, v)$ given by (16).

$$
\widehat{C}(u, v)=\frac{\mathcal{F}^{*}(u, v) \overline{\mathcal{F}(u, v)}}{\left|\mathcal{F}^{*}(u, v) \overline{\mathcal{F}(u, v)}\right|}=e^{j 2 \pi\left\{\frac{u}{M} \delta_{x}+\frac{v}{N} \delta_{y}\right\}}
$$

where, $\overline{\mathcal{F}(u, v)}$ is the complex conjugate of $\mathcal{F}(u, v)$. It is normalized in order to compensate the intensity variations. Now, (16) can be solved for overall translation $\left(\delta_{x}, \delta_{y}\right)$. The convenient way that is used in this work is to find the inverse Fourier transform of (16) that results in a Dirac delta function given by (17).

$$
\mathcal{D}\left(\delta_{x}, \delta_{y}\right)=\mathcal{F}^{-1}(\widehat{C}(u, v))
$$

Finally, the 2D translation is computed by finding the maximum of (17).

$$
\left(\tau_{x}, \tau_{y}\right)=\operatorname{argmax}\left\{\mathcal{D}\left(\delta_{x}, \delta_{y}\right)\right\}
$$

where, $\tau_{x}$ and $\tau_{y}$ are the estimated translations in $x$ and $y$ directions, respectively. Similar to the translation, the rotation can also be estimated by correlating the magnitude spectra. However, it is not described in this paper since the platform can be controlled only in $x$ and $y$ directions. 
2) Control scheme: For positioning the platform, a control scheme has been designed considering the $2 \mathrm{D}$ translation as the visual features computed from the previous step. If in case, the rotation is used, the resulting control can allow us to decouple the control of rotation and translation (in our case only translation is used). The current visual features are

$$
\mathbf{s}(t)=\left[\mathbf{s}_{v}\right]^{\top}
$$

where, $\mathbf{s}_{v}=\left[\begin{array}{lll}\tau_{x} & \tau_{y}\end{array}\right]^{\top}$ are the translations in $x, y$ and $z$ (=0) axes. The final objective is to drive the platform to the desired location i.e. $\mathbf{s}^{*}=\left[\mathbf{0}_{1 \times 3}\right]^{\top}$. The task function (error) e to be regulated is then given by

$$
\mathbf{e}=\mathbf{e}_{v}=\mathbf{s}-\mathbf{s}^{*}=\left[\begin{array}{lll}
\tau_{x} & \tau_{y} & 0
\end{array}\right]^{\top}
$$

In this case, the shape of the task function that has been computed offline by considering only $2 \mathrm{D}$ translation is shown in the Fig. 3(b). By comparing the shape of cost functions given by both methods, it is clear that the Fourier-based method shows better convergence. This is mainly due to its robustness to intensity variations. Now, the relationship between time variation of visual features and the camera instantaneous velocities is given by (21).

$$
\dot{\mathbf{s}}=\dot{\mathbf{s}}_{v}=\mathbf{L}_{v} \mathbf{v}_{v(3 \times 1)}
$$

where, $\mathbf{L}_{v}$ is a $3 \times 3$ interaction matrix (identity) to link the linear velocity. By considering the exponential convergence of the error i.e. $\dot{\mathbf{e}}=-\lambda \mathbf{e}$, where $\lambda$ is a positive gain value, the control law is then given by

$$
\mathbf{v}=-\lambda_{v} \mathbf{L}_{v} \mathbf{e}_{v}
$$

where, $\lambda_{v}$ is positive translational gain. The final control used is

$$
\left[\begin{array}{l}
v_{x} \\
v_{y}
\end{array}\right]=-\lambda_{v}{ }^{c} \mathbf{V}_{p} \mathbf{L}_{v}{ }^{-1}\left[\begin{array}{l}
\tau_{x} \\
\tau_{y}
\end{array}\right]
$$

where, $v_{x}$ and $v_{y}$ are the platform velocities in $x$ and $y$ directions, respectively. Later, similar to the intensity-based visual servoing, the displacements and voltages are computed using (11), (12), (1), (2), (3) and (4).

\section{EXPERIMENTAL VALIDATIONS}

Positioning has been performed on silicon microparts (see Fig. 4) of dimensions $10 \mu m \times 100 \mu m \times 20 \mu m$ placed on the micropositioning stage. The task is to position the parts automatically in the desired location by controlling the platform's 2 degrees of freedom ( $x y$ plane). For demonstration, the desired location has been selected by an operator using GUI. Once it is selected, the platform moves back to its initial position $(-19 \mathrm{~V},-19 \mathrm{~V})$ from where the servoing starts. Different experimental conditions that include optimal scan speed, high scan speed and high magnification are tested for both methods. For all tests, the SEM secondary electron images of size $512 \times 512$ pixels are used. Here after, we call intensity-based method as method-1 and Fourier-based method as method-2 for simplicity.

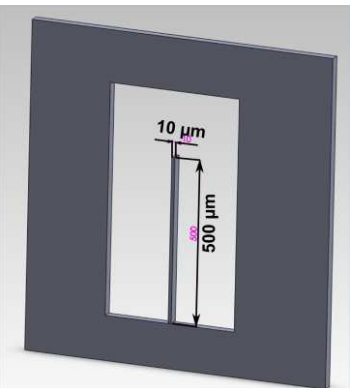

Fig. 4. Silicon micropart used for the experiments.

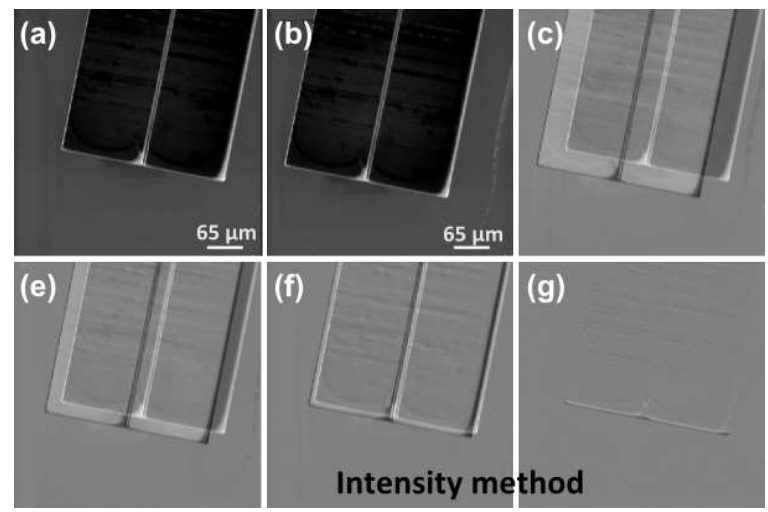

Fig. 5. Series of images depicting method-1 at optimal scan speed. (a) Selected desired position. (b) Initial image. (c) - (f) Errors at different positions. (g) Final error.

\section{A. Positioning task using the images acquired with optimal scan speed}

The initial experiments are conducted to perform a positioning task and to validate the proposed methods with an optimal scan speed of 720 nanoseconds per pixel. The magnification is fixed to $300 \times$. For method-1, the user selected desired voltages are $50 \mathrm{~V}$ for $x$-channel and $60 \mathrm{~V}$ for $y$-channel. Fig. 5(a) and Fig. 5(b) show respectively the images acquired at desired and initial positions for method-1. Fig. 5(c) to Fig. 5(f) show error $\left(\mathbf{I}-\mathbf{I}^{*}\right)$ at different locations. Fig. $5(\mathrm{~g})$ shows final error at the end of positioning task. Platform displacement and cost variations during this test are shown in Fig. 6(a) and Fig. 6(b) respectively. Similarly, for method-2, an initial voltage of $50 \mathrm{~V}$ has been selected for both channels and Fig. 7(a) shows the image acquired at this location. Fig. 7(b) shows initial image in visual servoing process and Fig. 7(c) to Fig. 7(f) show the subtracted images of reference and current images (visual representation of error) during the process. Fig. 7(g) shows the error at final location. The displacement and error variations during the

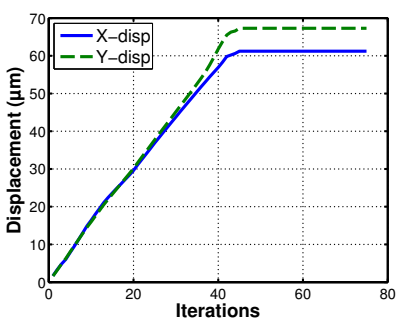

(a)

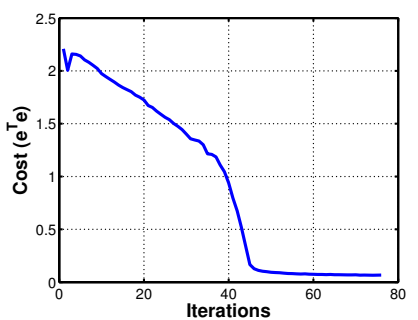

(b)
Fig. 6. (a) Displacement (b) cost variations during the positioning task with method-1 using optimal scan speed. 

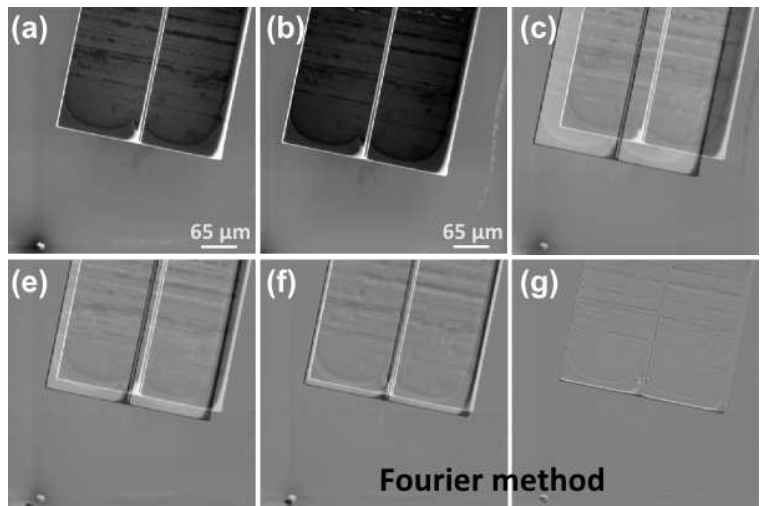

Fig. 7. Sequence of images acquired with method- 2 at optimal scan speed. (a) Desired location. (b) Initial image in the process. (c) - (f) Errors at different positions. (g) Final error.

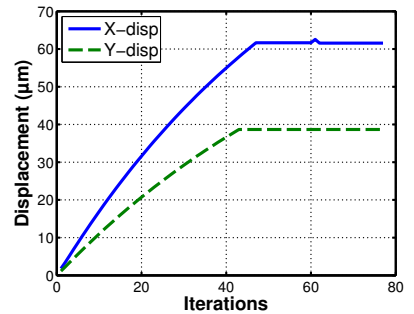

(a)

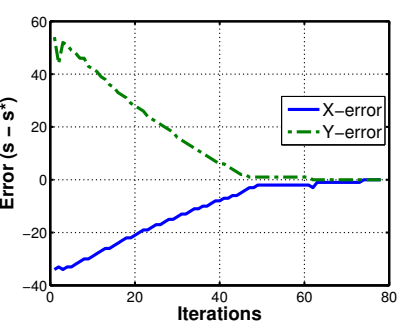

(b)
Fig. 8. (a) Displacement (b) error variations with method-2 using optimal scan speed.

process are shown in Fig. 8. From the obtained results, it can be seen that both the methods succeeded in reaching the desired position; however, method- 2 shows better accuracy.

\section{B. Positioning task using the images acquired with increased scan speed}

Second tests are performed with an increased raster scanning speed where the image noise is more. It has been performed to check the method's efficiency in reaching the desired position at noisy conditions. For this test a scan speed of 360 nanoseconds per pixel (maximum allowed) has been used. The selected desired voltages for method1 are $50 V$ and $60 V$ for $x$ and $y$ channels respectively. Fig. 9(a) and Fig. 9(b) show the initial image and initial error during the visual servoing process and Fig. 9(c) shows the final error. Displacement and cost variations during this test are shown in Fig. 11(a) and Fig. 11(b) respectively. For method-2, $40 \mathrm{~V}$ and $50 \mathrm{~V}$ are selected respectively for $x$ and $y$ channels. Fig. 10(a), Fig. 10(b) and Fig. 10(c) show respectively the initial image, initial error and final error during the visual servoing process using method-2. The displacement and error variations are shown in Fig. 12. From
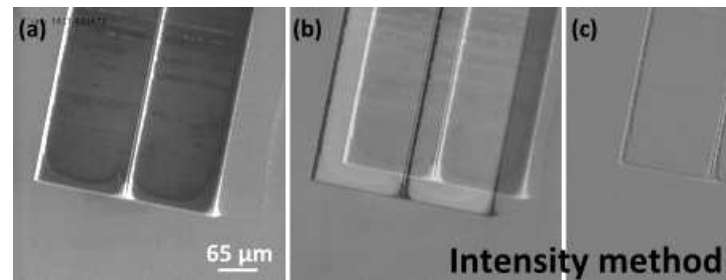

Fig. 9. (a) Initial image (c) error at initial position (d) error at final position during the nanopositioning task using method-1 at high scan speed.

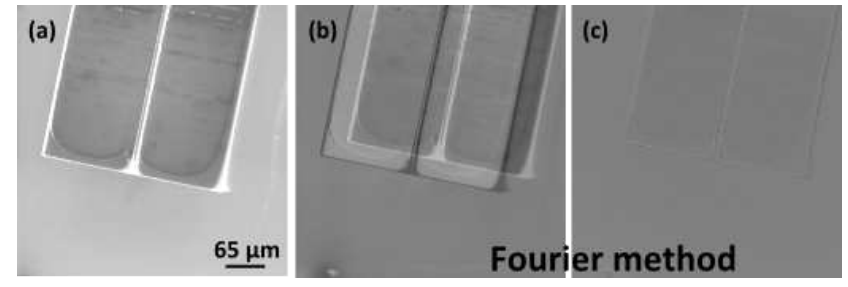

Fig. 10. (a) Image acquired at initial position (b) initial error (c) error during the nanopositioning task using method-2 at high scan speed.

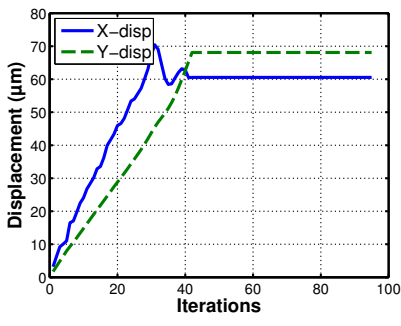

(a)

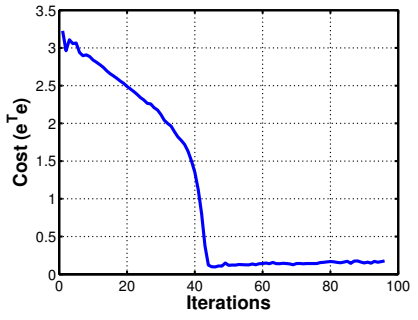

(b)
Fig. 11. (a) Displacement (b) cost variations during the positioning task with method-1 using high scan speed.

the obtained results, despite having high image noise, both methods reached the desired location. However, similar to the previous test, method- 2 shows better performance.

\section{Positioning task with high magnification}

Final experiments are conducted to perform the positioning task at a high magnification of $800 \times$. Simultaneously, both methods are also validated with increased scan speed at the selected magnification. The selected scan time is 360 nanoseconds per pixel. The desired voltages selected for method-1 are $30 V$ and $60 V$ respectively for $x$ and $y$ channels. Fig. 13(a), Fig. 13(b) and Fig. 13(c) show the initial image, error at initial position and final error respectively. Displacement and cost variations during this test are shown in Fig. 15(a) and Fig. 15(b) respectively. For method-2, the selected voltages are $40 \mathrm{~V}$ and $50 \mathrm{~V}$ respectively for $x$ and $y$ channels. Fig. 14(a), Fig. 14(b) and Fig. 14(c) show the initial

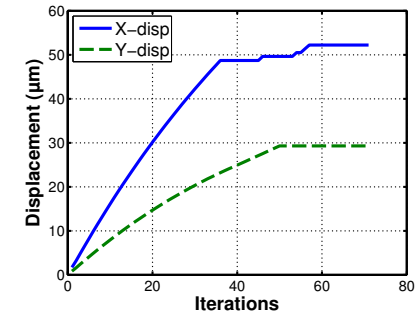

(a)

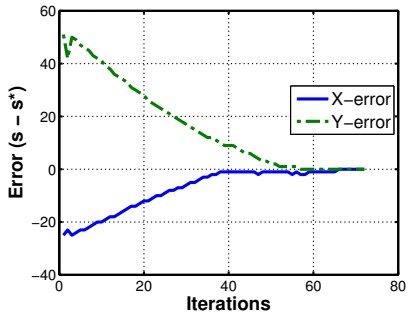

(b)
Fig. 12. (a) Displacement (b) error variations with method-2 using high scan speed for image acquisition.
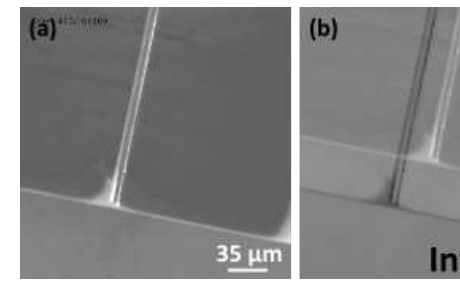

Fig. 13. (a) Image at initial position (b) initial error (c) final error during the nanopositioning task using method-1 at high magnification $(800 \times)$. 


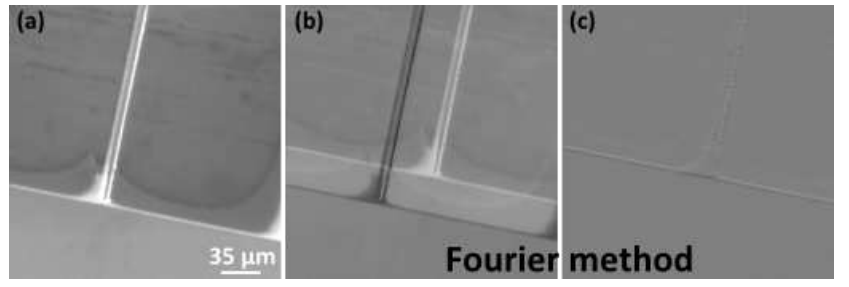

Fig. 14. (a) initial image (b) initial error (c) final error during the nanopositioning task using method-2 at high magnification $(800 \times)$.

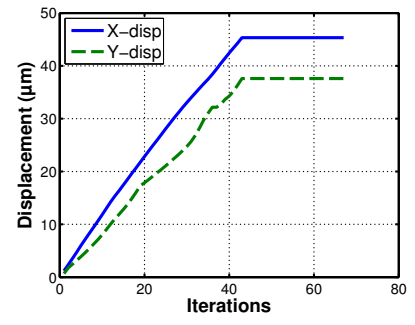

(a)

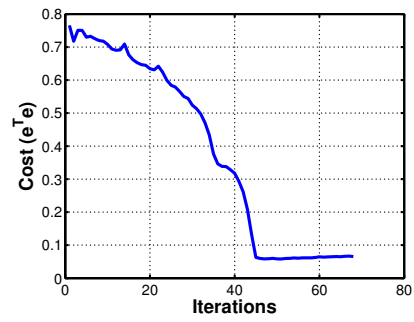

(b)
Fig. 15. (a) Displacement (b) cost variations during the positioning task with method-1 using high magnification.

image, error at initial position and final error respectively. The displacement and error variations are shown in Fig. 16.

The obtained results clearly demonstrate that the positioning task has been successfully accomplished at high magnification.

\section{Accuracy of positioning}

Since the existing system (SEM) does not allow using any external displacement measuring devices like laser interferometers, in this work, the positioning accuracy has been measured directly from the images. Eventhough, the estimated accuracy is not reliable (due to the presence of noise); it has been computed to demonstrate the efficiency of the methods in performing a nanopositioning task. Besides, it depends on the magnification used (from (24)) and increases with increase in the magnification. The accuracy is estimated using the final error image by multiplying the number of error pixels with pixel dimension on the sample. The pixel dimension $P$ on the sample is computed using (24).

$$
P=\frac{D}{G} \quad[\mu m]
$$

where, $D$ is the pixel dimension on the screen (constant) and $G$ is the magnification. With our system, the computed $D$ value is 212.3 for a screen size of $512 \times 512$ pixels. The accuracies computed with different tests demonstrated above

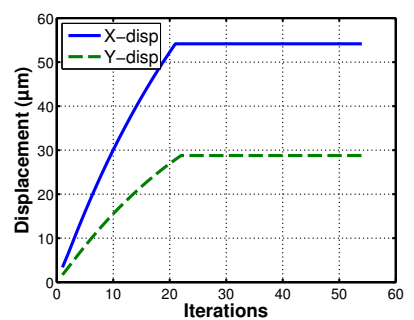

(a)

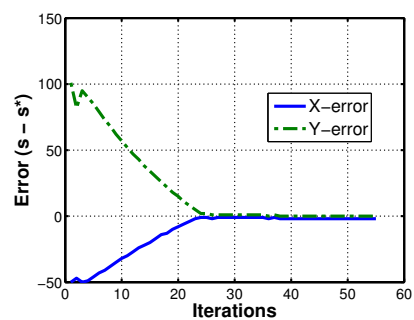

(b)
Fig. 16. (a) Displacement (b) error variations with method-2 at high magnification $(800 \times)$.
TABLE I

ESTIMATED POSITIONING ACCURACY $(\mu m)$ ACHIEVED BY BOTH METHODS.

\begin{tabular}{|c|c|c|c|c|}
\hline \multirow{2}{*}{ Condition } & \multicolumn{2}{|c|}{ Method-1 } & \multicolumn{2}{c|}{ Method-2 } \\
\cline { 2 - 5 } & $\mathrm{x}(\mu \mathrm{m})$ & $\mathrm{y}(\mu \mathrm{m})$ & $\mathrm{x}(\mu \mathrm{m})$ & $\mathrm{y}(\mu \mathrm{m})$ \\
\hline Optimal speed & 0.707 & 1.414 & 0.707 & 0.707 \\
\hline High scan rate & 1.061 & 2.123 & 0.707 & 0.707 \\
\hline High magnification & 0.398 & 0.5307 & 0.265 & 0.265 \\
\hline
\end{tabular}

are summarized in table I. The obtained results clearly show that method-2 provides good accuracy in positioning.

\section{CONCLUSION}

An automatic nanopositioning task of silicon microstructures using a SEM has been presented in this work. It has been accomplished using two approaches of visual servoing. The first method is a photometric approach where all the gray level intensities of an image are used as visual features. The error variation and platform movement are directly linked with the intensity variation. The second method is based on estimating the 2D motion between images using image frequency spectral information. Both the methods are validated at different experimental conditions. Even though both methods succeeded in accomplishing the overall task, Fourier-based method shows better behaviour and accuracy. This is mainly due to its nature of robustness to image noise and intensity variations (that is high in case of SEM imaging). The future work will concentrate on using the developed approaches for positioning a 3 degrees of freedom micromanipulator containing a microgripper to perform a complete nanomanipulation task.

\section{REFERENCES}

[1] K. Aoki, H. T. Miyazaki, H. Hirayama, K. Inoshita, T. Baba, K. Sakoda, N. Shinya, and Y. Aoyagi, "Microassembly of semiconductor three-dimensional photonic crystals," Nature materials, vol. 2, no. 2, pp. 117-121, 2003.

[2] B. Tamadazte, E. Marchand, S. Dembélé, and N. Le Fort-Piat, "Cad model-based tracking and $3 \mathrm{~d}$ visual-based control for mems microassembly," The International Journal of Robotics Research, vol. 29, no. 11, pp. 1416-1434, 2010.

[3] J. Agnus, D. Hériban, M. Gauthier, and V. Pétrini, "Silicon endeffectors for microgripping tasks," Precision engineering, vol. 33, no. 4, pp. 542-548, 2009.

[4] D. Jasper and S. Fatikow, "Automated high-speed nanopositioning inside scanning electron microscopes," in IEEE Conference on automation science and engineering, Aug 2010, pp. 704-709.

[5] S. Hutchinson, G. D. Hager, and P. I. Corke, "A tutorial on visual servo control," IEEE Transactions on Robotics and Automation, vol. 12, no. 5, pp. 651-670, 1996.

[6] B. Thuilot, P. Martinet, L. Cordesses, and J. Gallice, "Position based visual servoing: keeping the object in the field of vision," in Proceedings of the IEEE International Conference on Robotics and Automation. ICRA'02., vol. 2. IEEE, 2002, pp. 1624-1629.

[7] F. Chaumette and S. Hutchinson, "Visual servo control. i. basic approaches," IEEE Robotics \& Automation Magazine, vol. 13, no. 4, pp. 82-90, 2006.

[8] B. Tamadazte, G. Duceux, N.-F. Piat, and E. Marchand, "Highly precise micropositioning task using a direct visual servoing scheme," in Proceedings of the IEEE International Conference on Robotics and Automation. ICRA'11. IEEE, 2011, pp. 5689-5694.

[9] E. Marchand, "Control camera and light source positions using image gradient information," in IEEE International Conference on Robotics and Automation. ICRA'07, 2007, pp. 417-422.

[10] E. Malis, "Improving vision-based control using efficient second-order minimization techniques," in Proceedings of the IEEE International Conference on Robotics and Automation. ICRA'04., vol. 2. IEEE, 2004, pp. 1843-1848. 